OPEN ACCESS

Edited by:

Tongjie Liu,

Ocean University of China, China

Reviewed by:

Takeshi Haneda,

Kitasato University, Japan

Kenneth James Genovese,

Agricultural Research Service,

United States Department

of Agriculture, United States

*Correspondence:

Parameth Thiennimitr

parameth.t@cmu.ac.th;

parameth@gmail.com

orcid.org/0000-0002-6339-8744

Specialty section:

This article was submitted to

Food Microbiology,

a section of the journal

Frontiers in Microbiology

Received: 29 May 2021 Accepted: 04 August 2021

Published: 23 August 2021

Citation:

Buddhasiri S, Sukjoi C, Kaewsakhorn T, Nambunmee $K$, Nakphaichit M, Nitisinprasert $S$ and Thiennimitr P (2021) Anti-inflammatory Effect of Probiotic Limosilactobacillus reuteri KUB-AC5 Against Salmonella Infection in a Mouse Colitis Model.

Front. Microbiol. 12:716761 doi: 10.3389/fmicb.2021.716761

\section{Anti-inflammatory Effect of Probiotic Limosilactobacillus reuteri KUB-AC5 Against Salmonella Infection in a Mouse Colitis Model}

\author{
Songphon Buddhasiri', Chutikarn Sukjoi', Thattawan Kaewsakhorn'2, \\ Kowit Nambunmee ${ }^{3,4}$, Massalin Nakphaichit ${ }^{5}$, Sunee Nitisinprasert ${ }^{5}$ and \\ Parameth Thiennimitr $1,6,7 *$
}

'Department of Microbiology, Faculty of Medicine, Chiang Mai University, Chiang Mai, Thailand, ${ }^{2}$ Department of Veterinary Biosciences and Veterinary Public Health, Faculty of Veterinary Medicine, Chiang Mai University, Chiang Mai, Thailand, ${ }^{3}$ Major of Occupational Health and Safety, School of Health Science, Mae Fah Luang University, Chiang Rai, Thailand, ${ }^{4}$ Urban Safety Innovation Research Group, Mae Fah Luang University, Chiang Rai, Thailand, ${ }^{5}$ Department of Biotechnology, Faculty of Agro-Industry, Kasetsart University, Bangkok, Thailand, ${ }^{6}$ Research Center of Microbial Diversity and Sustainable Utilization, Chiang Mai University, Chiang Mai, Thailand, ${ }^{7}$ Faculty of Medicine, Center of Multidisciplinary Technology for Advanced Medicine, Chiang Mai University, Chiang Mai, Thailand

Acute non-typhoidal salmonellosis (NTS) caused by Salmonella enterica Typhimurium (STM) is among the most prevalent of foodborne diseases. A global rising of antibiotic resistance strains of STM raises an urgent need for alternative methods to control this important pathogen. Major human food animals which harbor STM in their gut are cattle, swine, and poultry. Previous studies showed that the probiotic Limosilactobacillus (Lactobacillus) reuteri KUB-AC5 (AC5) exhibited anti-Salmonella activities in chicken by modulating gut microbiota and the immune response. However, the immunobiotic effect of AC5 in a mammalian host is still not known. Here, we investigated the anti-Salmonella and anti-inflammatory effects of AC5 on STM infection using a mouse colitis model. Three groups of C57BL/6 mice (prophylactic, therapeutic, and combined) were fed with $10^{9}$ colony-forming units (cfu) AC5 daily for 7, 4, and 11 days, respectively. Then, the mice were challenged with STM compared to the untreated group. By using a specific primer pair, we found that AC5 can transiently colonize mouse gut (colon, cecum, and ileum). Interestingly, AC5 reduced STM gut proliferation and invasion together with attenuated gut inflammation and systemic dissemination in mice. The decreased STM numbers in mouse gut lumen, gut tissues, and spleen possibly came from longer AC5 feeding duration and/or the combinatorial (direct and indirect inhibitory) effect of AC5 on STM. However, AC5 attenuated inflammation (both in the gut and in the spleen) with no difference between these three approaches. This study demonstrated that AC5 confers both direct and indirect inhibitory effects on STM in the inflamed gut.

Keywords: acute non-typhoidal salmonellosis, Salmonella enterica Typhimurium, probiotic Limosilactobacillus (Lactobacillus), mouse colitis model, anti-inflammatory effect, immunomodulation 


\section{INTRODUCTION}

Acute gastroenteritis caused by non-typhoidal Salmonella is a foodborne disease causing around 93.8 million cases and 155,000 deaths per year worldwide (Majowicz et al., 2010; Heredia and García, 2018; Balasubramanian et al., 2019). The Gram-negative bacterium Salmonella enterica is comprised of more than 2,500 serovars. The most important non-typhoidal serovars for human or livestock infections include Typhimurium, Enteritidis, Gallinarum, Infantis, Ohio, Seftenberg, Derby, and Rissen (Antunes et al., 2016; Campos et al., 2019; Ferrari et al., 2019; Sun et al., 2021). The major route of human acute nontyphoidal salmonellosis (NTS) is consumption of contaminated food or drink from animal reservoirs of Salmonella (especially cattle, swine, and chickens) (Bernad-Roche et al., 2021). Increased demand for food products in both developed and developing countries leads to the improper use of antibiotics on animal farms. Low dose antibiotics have been used as a growth promoter to increase the yield of meat products (Castanon, 2007). However, this practice results in an increase in multi-drug resistance (MDR) Salmonella strains around the world (Zhao and Hu, 2013; Ur Rahman et al., 2018; Sedrakyan et al., 2020). MDR Salmonella strains are currently spreading in the human food supply chain and have a huge impact on economics and health (Tasmin et al., 2019; Castro-Vargas et al., 2020; Chen et al., 2020). This concern raised the alarm on the need to find alternative ways to control this important foodborne pathogen (Kongsanan et al., 2020; Mao et al., 2021; Sargun et al., 2021).

Salmonella enterica serovar Typhimurium (STM) is a major cause of human acute NTS and its pathogenesis has been extensively investigated (Broz et al., 2012; Rivera-Chavez and Baumler, 2015; Hausmann and Hardt, 2019; Rogers et al., 2021). Once ingested and passed into the large intestine of its mammalian host, STM exploits two type-three secretion systems (T3SS)-1 and T3SS-2 to secrete effector proteins promoting gut epithelial invasion and survival inside host cells, respectively. Innate immune receptors such as Toll-like receptors (TLRs) or nucleotide-binding oligomerization domain-(NOD)like receptors (NLRs) of gut epithelium recognize pathogenassociated molecular patterns (PAMPs) on STM resulting in gut inflammation (Thiennimitr et al., 2012). Chemotactic cytokines such as interleukin (IL)-8 (CXCL- 8 or KC in mouse) and IL6 promote a neutrophil influx into the gut lamina propria and submucosal tissue at the site of STM entry (Barthel et al., 2003; Tsolis et al., 2011; Santos, 2014). A hallmark histological finding of STM infection in humans is infiltration in numerous polymorphonuclear cells (PMNs) in the large intestine. STM infection also increases the production of inducible nitric oxide synthase (iNOS) derived from gut epithelium and lamina propria inflammatory monocytes (Winter et al., 2013; McLaughlin et al., 2019). Then, iNOS catalyzes the production of nitric oxide (NO), which plays a significant role in the host immune response and Salmonella pathogenesis (Lopez et al., 2015). Interestingly, the facultative anaerobic STM can take advantage of gut inflammation to outcompete resident obligate anaerobic gut microbiota and bloom in the inflamed gut (Lupp et al., 2007; Stecher et al., 2007; Barman et al., 2008). In the inflamed gut, alternative terminal electron acceptors such as tetrathionate $\left(\mathrm{S}_{4} \mathrm{O}_{6}{ }^{2-}\right)$ and nitrate $\left(\mathrm{NO}_{3}{ }^{-}\right)$become available for STM. STM exploits these electron acceptors to breakdown non-fermentable molecules such as ethanolamine and 1,2propanediol by anaerobic respiration (Winter et al., 2010, 2013; Thiennimitr et al., 2011; Faber et al., 2017). This nutritional advantage allows STM to outcompete gut microbiota and flourish in the host gut during the infection (Rivera-Chavez and Baumler, 2015). Successful proliferation in the inflamed gut plays a crucial role in STM transmission to a new host (Stecher et al., 2007; Santos et al., 2009).

Probiotics are defined as "live microorganisms when administered in adequate amounts confer a health benefit on the host" (Morelli and Capurso, 2012). Several probiotics have already been investigated to identify potential beneficial roles against Salmonella infection. A Gram-positive bacterium in the genus Limosilactobacillus (former named "Lactobacillus") is one of the most common probiotic microorganisms used to promote animal and human health (Kakabadze et al., 2020; Petrova et al., 2021). Several species of Limosilactobacillus, for example, reuteri, paracasei, johnsonii, plantarum, and rhamnosus are categorized as generalized recognized as safe (GRAS) and can be used as probiotics. Previous studies have already demonstrated an anti-Salmonella effect of the probiotic Limosilactobacillus both in vitro and in vivo (Nakphaichit et al., 2019; Hai et al., 2021; Jia et al., 2021; Kim et al., 2021). Effects of probiotics against enteropathogens (colonization resistance) can be divided into two major branches: (1) direct effect including prevention of pathogen adhesion and invasion to gut epithelium, nutritional competition with pathogens, or direct killing activity on pathogens by the production of antimicrobial substances and (2) indirect effect by enhancing host protective immune response (locally or systemically) such as strengthening gut barrier integrity, increasing production of secretory IgA (SIgA) antibody or attenuation of inflammatory responses (Sassone-Corsi and Raffatellu, 2015). Several strains of Limosilactobacillus reduced Salmonella lipopolysaccharide (LPS)-induced gut epithelial barrier impairment by increasing tight junction protein expressions (Fang et al., 2010; Yeung et al., 2013). Limosilactobacillus probiotics attenuated Salmonellainduced gut inflammation by reducing pro-inflammatory cytokine production by the attenuation of TLRs activation in vitro (Kanmani and Kim, 2020). Kanmani and Kim (2020) showed that Limosilactobacillus isolated from Korean food increased production of the immunosuppressive cytokine IL-10, transforming growth factor (TGF)- $\beta$, and antimicrobial peptide $\beta$-defensin by manipulating the expression of TLR negative regulators in human colonocytes.

Liu et al. (2019) demonstrated anti-Salmonella activity of Limosilactobacillus in mice. They found that L. plantarum ZS2058 and L. rhamnosus GG (LGG) reduced Salmonella pathogenicity and inflammatory response in a mouse typhoid model with a strain-specific mechanism. The probiotic L. plantarum ZS2058 increased fecal short chain fatty acid (propionic acid) and mucin levels in mouse colon, while LGG more strongly alleviated mouse gut inflammation. This study reported the anti-Salmonella activity of the probiotic Limosilactobacillus in mice. However, 
mice infected with STM do not properly develop a robust gut inflammation but rather have a systemic infection called "typhoid fever-like disease" (Tsolis et al., 1999, 2011). To study the antiinflammatory role of the probiotic Limosilactobacillus against an acute NTS, a mouse colitis model for Salmonella infection should be used. Antibiotic pretreatment in the genetically susceptible mouse strains (e.g., $\mathrm{C} 57 \mathrm{BL} / 6$ and $\mathrm{BALB} / \mathrm{c}$ ) prior to STM infection transiently abrogates mouse colonization resistance. Then, STM triggers mouse gut inflammation which mimics acute gastroenteritis in human (Bohnhoff et al., 1954; Bohnhoff and Miller, 1962).

The anti-Salmonella effect of the probiotic AC5 has already been investigated in broiler chickens (Nakphaichit et al., 2011, 2019; Sobanbua et al., 2019). Nakphaichit et al. (2019) recently reported that oral feeding with AC5 increases the survival rate and attenuates the pathogenicity of Salmonella infection in chickens by modulating chicken gut microbiota and immune responses. Due to the differences in the gut immune response between avian and mammalian hosts when interacting with STM, the outcomes of probiotic Limosilactobacillus challenge between these two hosts might differ (Tsolis et al., 1999; Santos et al., 2001). Here, we investigated the anti-Salmonella and anti-inflammatory effects of the probiotic L. reuteri KUB-AC5 in the inflamed gut of STM-infected mice.

\section{MATERIALS AND METHODS}

\section{Ethical Approval}

The animal experiments in this study were approved by the Animal Care and Use Committee, Chiang Mai University, Thailand in accordance with the Association for Assessment and Accreditation of Laboratory Animal Care (AAALAC) guidelines (Approval No. 2559/MC-0005).

\section{Bacterial Strains, Culture Conditions, and Direct Antimicrobial Effect Assays}

The S. Typhimurium strain IR715 (STM) is a fully virulent, nalidixic acid-resistant derivative of the wild-type isolate ATCC 14028 (Stojiljkovic et al., 1995). The probiotic L. reuteri KUBAC5 (AC5) was isolated from chicken intestine (Nitisinprasert et al., 2000). To determine the direct anti-Salmonella activity of AC5, we performed growth assays in both liquid (co-culture) and solid media (spot-on lawn and agar-well diffusion assays). For the co-culture assay, $100 \mu \mathrm{L}$ of 1:100 dilution of an equal amount (1:1) of STM and AC5 inocula [about $10^{4}$ colonyforming unit $(\mathrm{cfu}) / \mathrm{mL}$ total] was incubated microaerobically (without shaking) in $10 \mathrm{~mL}$ of co-culture broth at $37^{\circ} \mathrm{C}$ for $16 \mathrm{~h}$ as previously described with a slight modification (Drago et al., 1997; Adetoye et al., 2018). The co-culture broth was composed of an equal amount of the double strength $(2 \times)$ de Man, Rogosa, and Sharpe (MRS) and Mueller-Hinton (MH) broth. For a single growth assay, $100 \mu \mathrm{L}$ of 1:100 dilution of an overnight culture of AC5 or STM was inoculated separately into $10 \mathrm{~mL}$ fresh MRS and $\mathrm{MH}$ broth, respectively, and then incubated at $37^{\circ} \mathrm{C}$ for $14 \mathrm{~h}$ without shaking. At the indicated time point, viable counts $(\mathrm{cfu} / \mathrm{mL})$ of AC5 and STM were enumerated using a serial 10-fold dilution with the appropriate media MRS agar (AppliChem) and Luria-Bertani (LB) agar with $0.05 \mathrm{mg} / \mathrm{mL}$ nalidixic acid, respectively.

The direct anti-Salmonella effect of the viable cell and cellfree supernatant from AC5 culture (AC5-CFS) of probiotic AC5 was determined by spot-on lawn and agar well diffusion methods as described previously (Lima et al., 2007). For the spot-on lawn assay, $20 \mu \mathrm{L}$ of AC5 overnight culture was spotted on MRS agar and incubated at $37^{\circ} \mathrm{C}$ for $16 \mathrm{~h}$ to develop a viable spot on the plate. Then, $200 \mu \mathrm{L}$ of STM overnight culture was added to $20 \mathrm{~mL} \mathrm{LB}$ broth containing 0.75\% agar (Difco agar, $\mathrm{BD}, \mathrm{MD}$, United States) and poured over the plate. All plates were incubated at $37^{\circ} \mathrm{C}$ for $16 \mathrm{~h}$ to observe the inhibition zone. A diameter of the zone of inhibition more than $1 \mathrm{~mm}$ was considered as a positive.

An agar well diffusion method was used to evaluate the antimicrobial effect of AC5-CFS on STM as previously described (Lima et al., 2007). To collect AC5-CFS, $5 \mathrm{~mL}$ of AC5 overnight culture was centrifuged at 4,000 rounds per minute (rpm) for $10 \mathrm{~min}$ at $4^{\circ} \mathrm{C}$. An STM lawn was prepared by mixing $200 \mu \mathrm{L}$ of STM overnight culture in $20 \mathrm{~mL} \mathrm{LB}$ with $0.75 \%$ agar and then pouring into a sterile plate. Then, 6-mm-diameter wells were made in the agar by $200 \mu \mathrm{L}$ pipette tip and filled with $60 \mu \mathrm{L}$ of AC5-CFS. All plates were incubated at $37^{\circ} \mathrm{C}$ for $16 \mathrm{~h}$ to observe the inhibition zone. The inhibition activity was expressed as a clear zone.

\section{Probiotic L. reuteri KUB-AC5 Preparation}

A single fresh colony of the probiotic L. reuteri KUB-AC5 grew on MRS agar (Difco agar, BD, MD, United States) with $0.6 \% \mathrm{w} / \mathrm{v}$ $\mathrm{CaCO}_{3}$ was picked and inoculated into $5 \mathrm{~mL}$ MRS broth with $0.6 \% \mathrm{w} / \mathrm{v} \mathrm{CaCO}_{3}$. The mixture was statically incubated at $37^{\circ} \mathrm{C}$ for 16-18 h. Then, $400 \mu \mathrm{L}$ AC5 overnight culture was added into a new $40 \mathrm{~mL}$ MRS broth with $0.6 \% \mathrm{w} / \mathrm{v} \mathrm{CaCO}_{3}$ (1:100 dilution) and grown statically for the next $16-18 \mathrm{~h}$. The bacterial culture was centrifuged at $4,000 \mathrm{rpm}$ at $4^{\circ} \mathrm{C}$ for $10 \mathrm{~min}$ to obtain the cell pellets which were resuspended with sterile $\mathrm{PBS}$ to adjust the final concentration of $10^{10} \mathrm{cfu} / \mathrm{mL}$ and kept at $4^{\circ} \mathrm{C}$ until use.

\section{Animal Study}

The animal experiments were approved by the Animal Care and Use Committee, Chiang Mai University, Thailand in accordance with the AAALAC guidelines (Approval No. 2559/MC-0005). Female C57BL/6 mice aged 6-8 weeks old were purchased from Nomura Siam International (Bangkok, Thailand). All mice were acclimatized and co-housed (for gut microbiota normalization) for 1 week and housed in 12 dark/12 light hour cycle in a controlled environment (room temperature, $21 \pm 1^{\circ} \mathrm{C}$; humidity, $50 \pm 10 \%)$. Standard mouse chow and drinking water were provided ad libitum. Then, mice were divided into four groups: (1) prophylactic (Pro), (2) therapeutic (Tx), (3) combined (prophylactic and therapeutic, Pro $+\mathrm{Tx}$ ), and (4) untreated control group. There were six to seven mice per group. Mouse was orally fed with $100 \mu \mathrm{L}$ of $10^{10} \mathrm{cfu} / \mathrm{mL}$ AC5 $\left(10^{9} \mathrm{cfu} / \mathrm{mouse}\right)$ daily for 7, 4, and 11 days for the prophylactic, therapeutic, and combined group, respectively. Mice in the untreated control group were daily fed with sterile PBS. To induce colitis, $100 \mu \mathrm{L}$ 
of (200 mg/mL) streptomycin sulfate (Sigma Aldrich, Singapore) solution was given orally to mice 1 day prior to STM infection ( $10^{9}$ cfu of STM/mouse) (Barthel et al., 2003; Sarichai et al., 2020). All mice were euthanized at day 4 post infection.

\section{Determination of Bacterial Numbers}

A viable count (cfu/gm tissue) of STM in mouse gut contents (colon content and cecal content), gut tissues (colon, cecum, and ileum), and spleen was performed using a serial-dilution plating technique. Briefly, the samples were collected in sterile PBS and homogenized with a bead-beating machine with $1.0 \mathrm{~mm}$ diameter zirconium/silica beads (Biospec Products, Bartlesville, OK, United States). Then, serial 10-fold dilutions of the homogenates were made. STM cfu/gm tissues were enumerated by plating $100 \mu \mathrm{L}$ of the dilutions onto LB agar with nalidixic acid $(0.05 \mathrm{mg} / \mathrm{mL})$ and incubated at $37^{\circ} \mathrm{C}$ for $16 \mathrm{~h}$.

Limosilactobacillus reuteri KUB-AC5 was quantified by quantitative polymerase chain reaction (qPCR) with the AC5 strain-specific primer pair (Sobanbua et al., 2019). The forward and reverse primers were TCGCTCACGGCTGTTAGGACA and AGCACTCCACGTTGCCACA, respectively. The microbial genomic DNA was extracted from mouse gut contents by using the QIAamp Stool Mini Kit (Qiagen, Hilden, Germany) in a combination with the bead beating method. About 100$200 \mathrm{mg}$ of gut contents was suspended in $900 \mu \mathrm{L}$ of sterile PBS (pH 8.0), $300 \mu \mathrm{L}$ phenol-chloroform-isoamyl alcohol (25:24:1) and $0.3 \mathrm{~g}$ of zirconium beads $(0.1 \mathrm{~mm}$ in diameter, As One Corporation, Osaka, Japan). Microbial cells in gut contents were broken by a minibead beater 3110BX (Biospec, United States) for $3 \mathrm{~min}$ at 4,800 rpm and further DNA extraction was done in accordance with the protocol of the QIAamp Stool Mini Kit. Total bacterial DNA was applied as a template for qPCR. Total qPCR reaction volume of $20 \mu \mathrm{L}$ contained $50 \mathrm{ng} / \mu \mathrm{L}$ of DNA template, $2 \mu \mathrm{L} ; 10 \mu \mathrm{mol}$ of each forward and reverse primer, $0.4 \mu \mathrm{L}$; LightCycler 480 SYBR Green master (Roche, Germany), $10 \mu \mathrm{L}$; and nuclease-free water added to obtain the final volume of $20 \mu \mathrm{L}$. Amplification conditions of initial denaturation at $95^{\circ} \mathrm{C}$ for 5 min were followed by 45 cycles of $95^{\circ} \mathrm{C}$ for $10 \mathrm{~s}$, annealing and extension step combined at $70^{\circ} \mathrm{C}$ for $45 \mathrm{~s}$, and elongation at $72^{\circ} \mathrm{C}$ for $5 \mathrm{~min}$. A serial 10 -fold dilution of genomic DNA of L. reuteri KUB-AC5 was used to generate the standard curve.

\section{Detection of Tissue Gene Expression by qPCR}

Four days after the infection with STM, all mice were euthanized and tissue samples were collected for mRNA expressions by qPCR. Fold change (relative of expression) of important proinflammatory cytokine genes [ $K c$, Ifng, and Il-6 encoding for KC or CXCL-1, interferon (IFN)-gamma and IL-6, respectively], Nos 2 and Zo-1 [encoding for iNOS and a tight junction protein zonula occludens (ZO-1), respectively], were performed as previously described (Sarichai et al., 2020). In short, collected tissues were immersed in RNA preservative solution (RNAstore reagent, TIANGEN, China) and stored at $-20^{\circ} \mathrm{C}$ until extraction. Mouse tissue RNA was isolated using TRIzol reagent (Thermo Fisher scientific, Waltham, MA, United States), following the manufacturer's protocol. DNase treatment was performed using a DNA removal and inactivation kit (Ambion, Life technologies, Vilnius, Lithuania). The purity and amount of the isolated RNA were confirmed using a NanoDrop Spectrophotometer (Thermo Fisher scientific, Waltham, MA, United States). Then, complimentary DNA (cDNA) was synthesized using a Tetro cDNA synthesis kit (Bioline, Taunton, MA, United States). The qPCR reaction was prepared by using a SensiFAST SYBR LoROX Kit (Bioline, Taunton, MA, United States) and performed with ViiA 7 Real-Time PCR system (Applied Biosystems). Gene expressions were analyzed with a comparative $C t$ method $\left(2^{-\Delta}\right.$ $\Delta C t$ ) using a Gapdh as a housekeeping genes (Schmittgen and Livak, 2008). The lists of primers used in this study are shown in Supplementary Table 1.

\section{Cecal Histopathological Study}

Segments of mouse ceca were fixed in $10 \%$ buffered formalin, embedded in paraffin, and stained with hematoxylin and eosin (H\&E). The slides were blindly scored by the veterinary pathologist using the criteria shown in Supplementary Table 2.

\section{Statistical Analysis}

The cfu/gm of STM and relative fold changes of mRNA were logarithmically transformed before statistical analysis. Analysis was carried out using a parametric test [one-way analysis of variance (ANOVA) with Tukey's multiple comparisons test]. The cecal histopathological scores were statistically tested using a Kruskal-Wallis test with multiple comparisons as previously described (Sarichai et al., 2020). $P<0.05$ was considered statistically significant; *, **, and *** indicate $P$-values $<0.05$, 0.01 , and 0.001 , respectively.

\section{RESULTS}

\section{Limosilactobacillus reuteri KUB-AC5 Exhibited Direct Antimicrobial Activity on Salmonella Typhimurium}

In a previous study, the strain AC5 produced antimicrobial peptide KAC5 of $4.7 \mathrm{kD}$ against $S$. Enteritidis S003 with bactericidal activity in its mode of action (Sobanbua et al., 2020). To investigate whether the AC5 can inhibit growth of STM IR715 used in this study, the time course growth assays of both monoculture and co-culture were carried out as shown in Figure 1. Co-culture assay demonstrated that probiotic AC5 can inhibit the growth of STM and maintain at $10^{5} \mathrm{cfu} / \mathrm{mL}$ (Figure 1A) during 8-14 h compared to that of $10^{7} \mathrm{cfu} / \mathrm{mL}$ in the monoculture assay (Figure 1B). From the spot-on lawn assay, we found the clear zone only around the viable colonies of AC5 but not CFS (Figure 1D). By the agar well diffusion assay, we observed the clear zone around the wells of both CFS and KAN (positive control) at $16 \mathrm{~h}$ after the inoculation but not the MRS (negative control) (Figure 1E). The diameters of clear zone in both assays are depicted in Supplementary Figure 2. These data indicated the direct antagonistic effect of both viable cells and CFS of AC5 on STM IR715. 

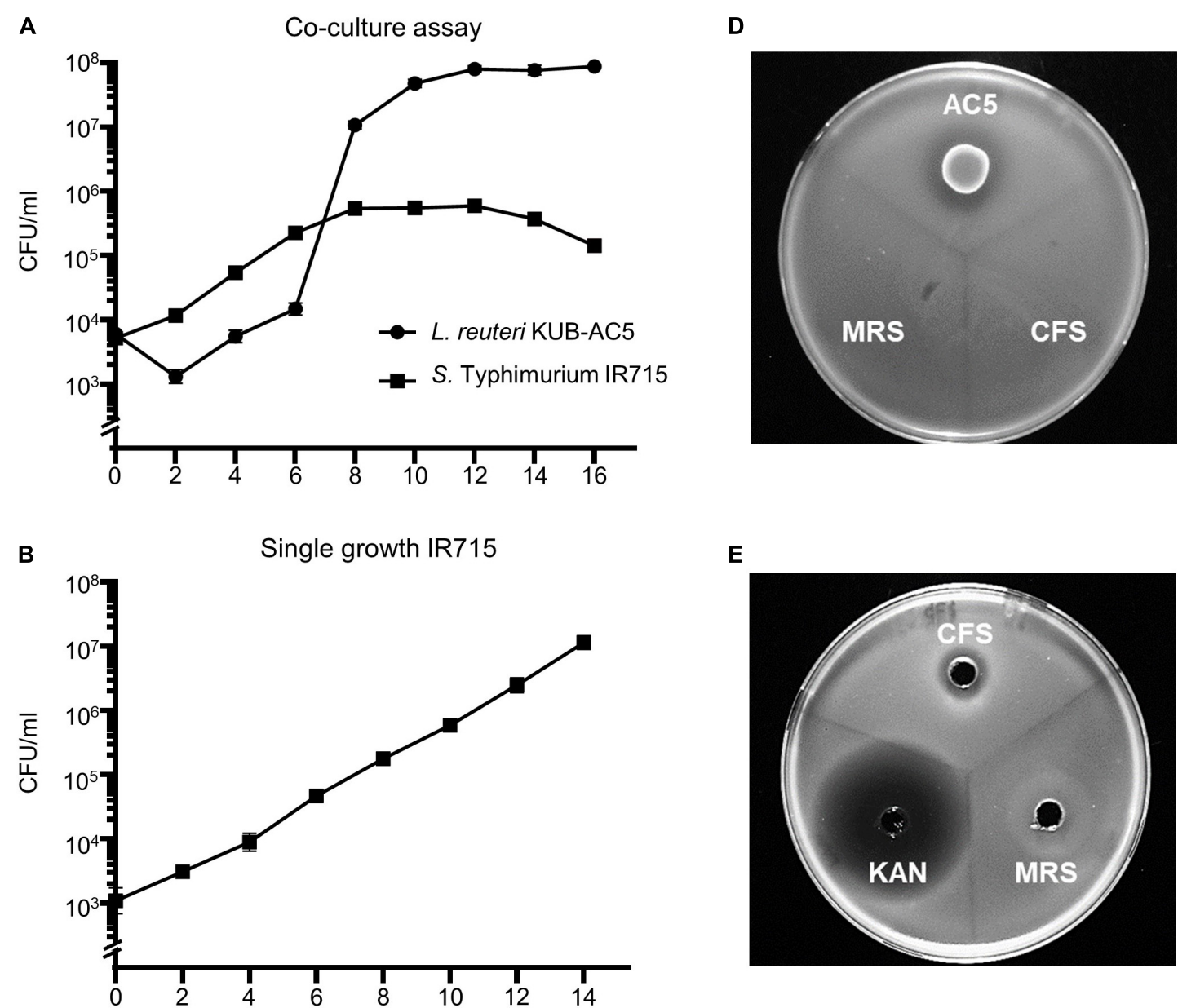

Single growth KUB-AC5

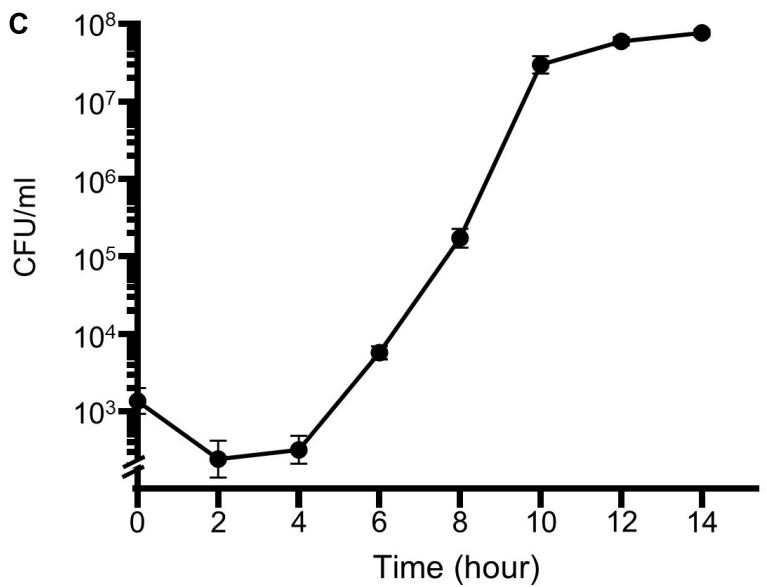

FIGURE 1 | In vitro anti-Salmonella effect of probiotic L. reuteri KUB-AC5 on S. Typhimurium IR715 in liquid and solid media. Co-culture assay was done by inoculating an equal amount of AC5 and STM in de Man, Rogosa, and Sharpe (MRS) and Mueller-Hinton (MH) broth (A). An independent growth of STM and AC5 in liquid media was performed (B,C, respectively). The anti-Salmonella activities of AC5 cell-free supernatant (CFS) were tested using spot-on lawn assay (D) and agar well diffusion assay (E). Data represent geometric means \pm SD of three independent experiments. KAN, kanamycin as a positive control, MRS broth as a negative control. 


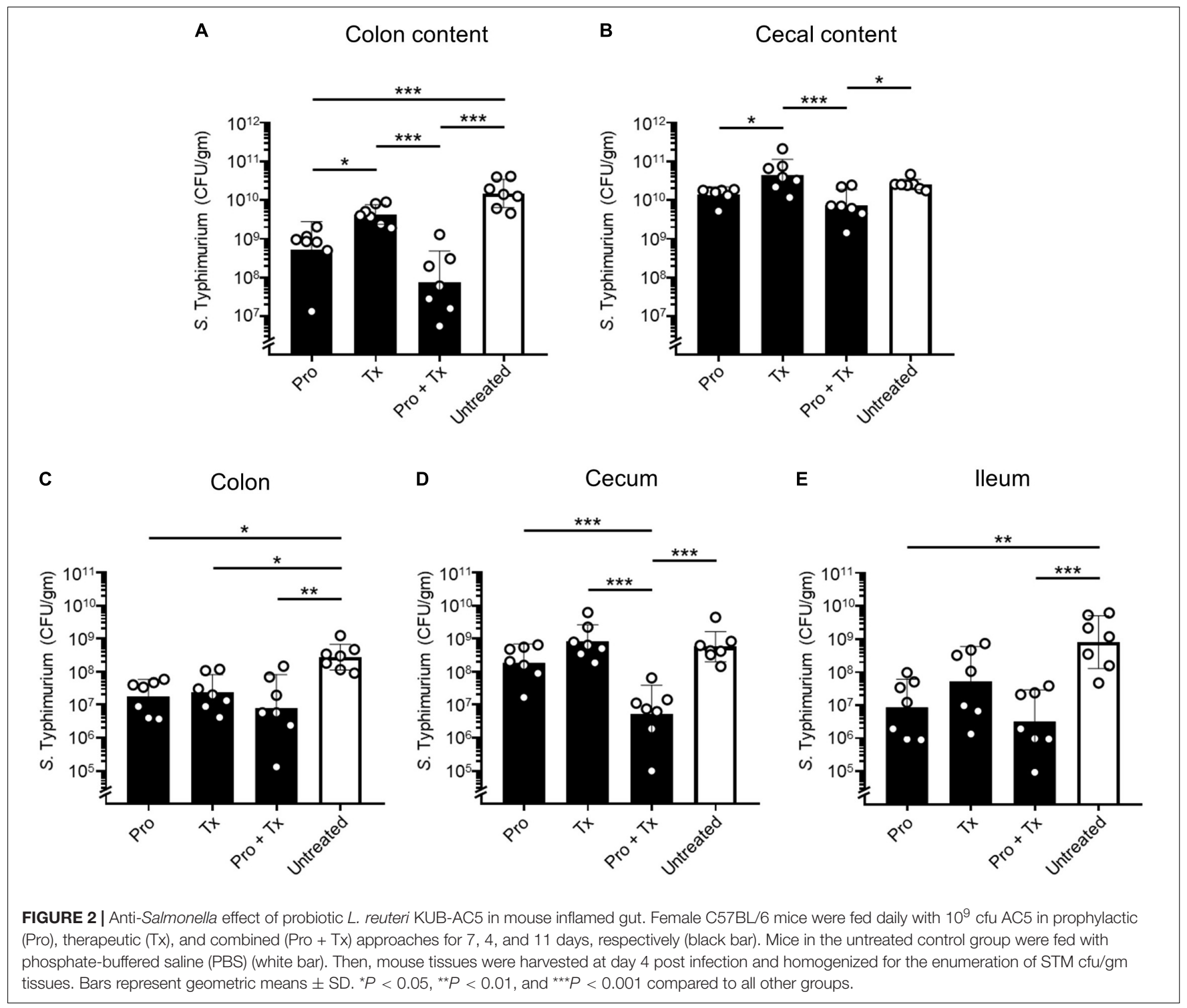

\section{Oral Feeding With L. reuteri KUB-AC5 Reduced Numbers of Salmonella in Mouse Inflamed Gut}

To study the role of AC5 consumption in an acute NTS, a mouse colitis model of STM infection was used. The 6-8week-old female C57BL/6 mice were divided into four groups: (1) prophylactic (Pro), (2) therapeutic (Tx), (3) combined (prophylactic and therapeutic, Pro $+\mathrm{Tx}$ ), and (4) untreated group. Mice in the treatment groups were orally fed daily with $10^{9} \mathrm{cfu}$ AC5 at the different indicated times (Supplementary Figure 1). At day 4 post infection, all mice were euthanized and tissue samples were harvested. Our data indicated that all groups of mice fed with AC5 have decreased numbers of STM in their gut contents (colon content and cecal content) (Figures 2A,B). Mice in the combined group (Pro $+\mathrm{Tx}$ ) showed a remarkable decrease in STM numbers in their colon contents. To evaluate the role of AC5 against STM gut tissue invasion, STM cfu/gm from different sites of the gut (colon, cecum, and ileum) were determined. Our data showed that all AC5-fed mice had significantly lower STM numbers in their gut tissues than those of the untreated mice (Figures 2C-E).

\section{The Probiotic L. reuteri KUB-AC5 \\ Transiently Colonized Mouse Gut}

To detect the presence of AC5 in mouse gut, we used a qPCR technique with a primer pair specific to AC5. Then, copy numbers of AC5 per mg of mouse gut content were calculated as previously described (Sobanbua et al., 2019). Our data demonstrated that AC5 can colonize differently in mouse colon, cecum, and ileum (Figure 3). Interestingly, the difference in AC5 feeding duration might play a role in a significant difference of AC5 amounts collected from mouse gut. In the therapeutic (Tx) and combined (Pro + Tx) groups, mice were orally fed with AC5 continuously for 4 and 11 days, respectively, before the sample 

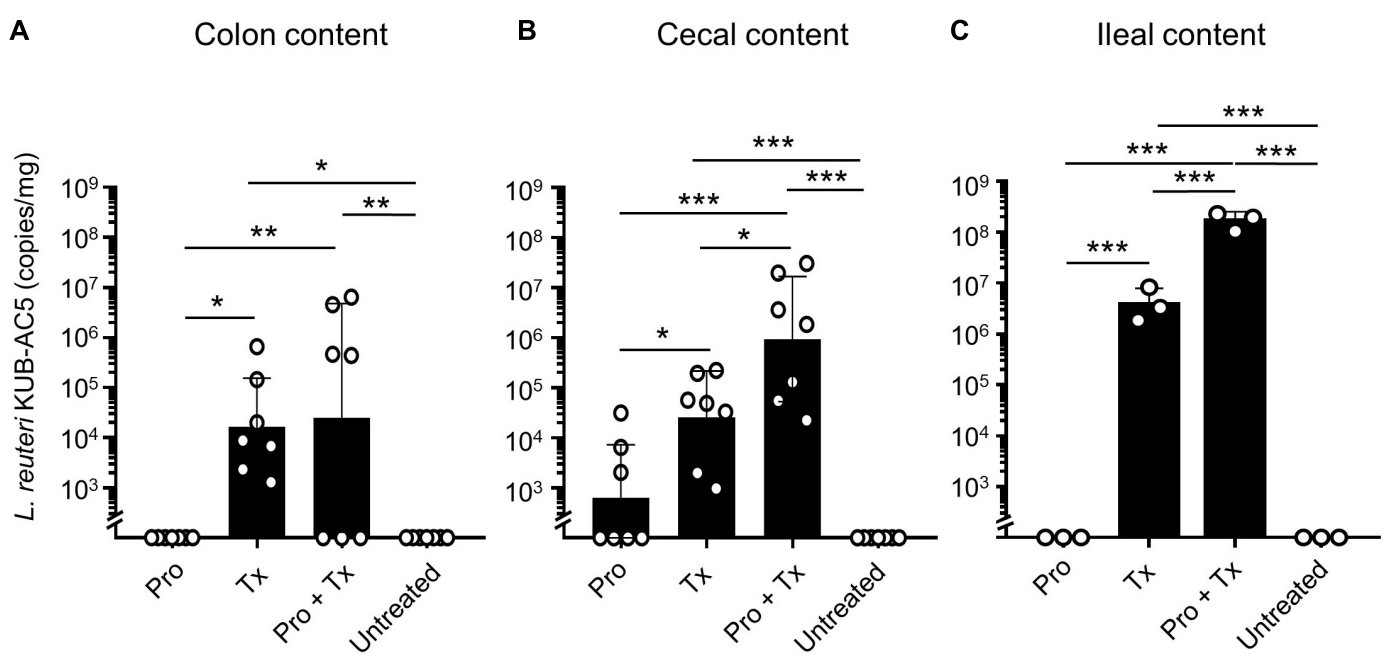

FIGURE 3 | Detectable amounts of $L$. reuteri KUB-AC5 in mouse gut contents. At day 4 post infection, mouse gut contents in colon, cecum, and ileum were collected and quantified for AC5 by qPCR. The copies/mg of AC5 in each gut content was calculated and depicted. Bars represent geometric means \pm SD. ${ }^{\star} P<0.05,{ }^{* \star} P<0.01$, and ${ }^{\star \star \star} P<0.001$ compared to all other groups.

collection. At the same time, mice in the prophylactic (Pro) group were fed with AC5 for 7 days and stopped for 4 days before the sample collection. Our results showed that mice in the prophylactic group have the lowest amount of AC5 in their gut contents. These data suggest the transient manner of AC5 colonization in mouse gut.

\section{The Probiotic L. reuteri KUB-AC5 Attenuated Gut Inflammation During Salmonella Infection}

Next, we investigated the anti-inflammatory effect of AC5 on mouse gut. Relative expressions of the representative proinflammatory genes were determined by qPCR. Our results revealed that all AC5-fed mice have reduced expression levels of colonic pro-inflammatory genes ( $\mathrm{Kc}, \mathrm{Il}-6$, and Nos2) (Figures 4A-C, respectively). However, the colonic gene expressions of Ifng encoding for cytokine IFN- $\gamma$, an important cytokine in the systemic phase of STM infection, were not changed in all groups (Figure 4D). Therapeutic (Tx) and combined (Pro + Tx) orally fed with the probiotic AC5 mice showed increased expression of tight junction protein zonula occludens gene (Zo-1) in the colon (Figure 4E).

STM-infected mice orally fed with AC5 also showed reduced gene expressions of $K c$ and $I l-6$ in their ceca (Figures 5A,B). There was no significant change in cecal Nos2 expression in all groups of mice (Figure 5C). Mice in the prophylactic (Pro) and therapeutic (Tx) groups showed a reduction in Ifng gene expression (Figure 5D). Zo-1 was upregulated in the prophylactic and therapeutic groups (Figure 5E). An anti-inflammatory effect of AC5 in the distal ileum of STM-infected mice was also observed (Figure 6). Probiotic AC5 feeding decreased $K c$, Il6, Nos2, Ifng, and increased Zo-1 expressions in the ilea of the mice. To confirm the anti-inflammatory effect of AC5 on gut inflammation, tips of mouse ceca were sectioned, stained, and evaluated. The histological study revealed that AC5 feeding attenuated the severity of gut inflammation induced by STM compared to that of the untreated mice (Figure 7).

\section{The Probiotic L. reuteri KUB-AC5 Reduced Systemic Dissemination of Salmonella Typhimurium in Mice}

In the systemic phase of infection, STM survives inside the cytoplasmic vacuoles of host innate immune cells such as macrophages or monocytes. This intracellular niche protects STM from host immune recognition and allows STM to disseminate through lymphatic and blood vessels to distant organs such as the spleen (Broz and Monack, 2011; Broz et al., 2012). Our data showed that feeding mice with AC5 significantly reduced STM numbers in the spleen (Figure 8A). The combined regimen (Pro $+\mathrm{Tx}$ ) of AC5 provided the greatest effect in the reduction of the STM numbers in the mouse spleen. AC5 also decreased the expressions of Ifng and Nos 2 in mouse spleen (Figures 8B,C). These data indicated that orally fed with probiotic $L$. reuteri KUB-AC5 can reduce the severity the systemic phase of STM infection in mice.

\section{DISCUSSION}

STM is one of the most prevalent foodborne pathogens causing several million cases of acute gastroenteritis and invasive bacteremia in humans per year (Majowicz et al., 2010; Whistler et al., 2018). Although the most outcome of STM infection is self-limiting acute diarrhea, a life-threatening condition could occur in immunocompromised hosts such as those with human immunodeficiency virus (HIV)-infection, malnutrition, or extreme ages (Gordon et al., 2002; Preziosi et al., 2012). An increase in MDR STM necessitates an urgent alternative approach 


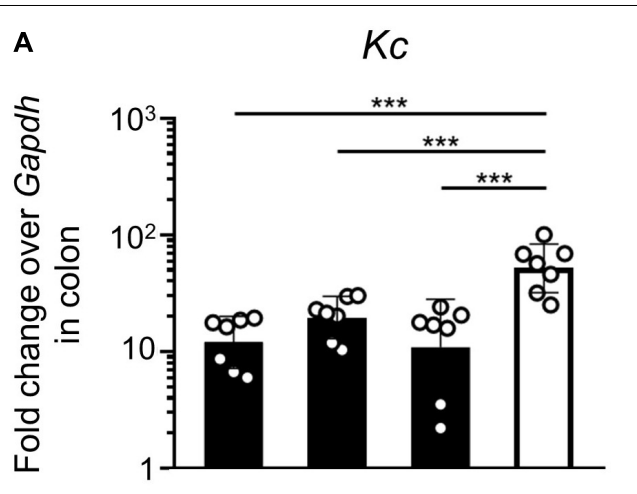

C

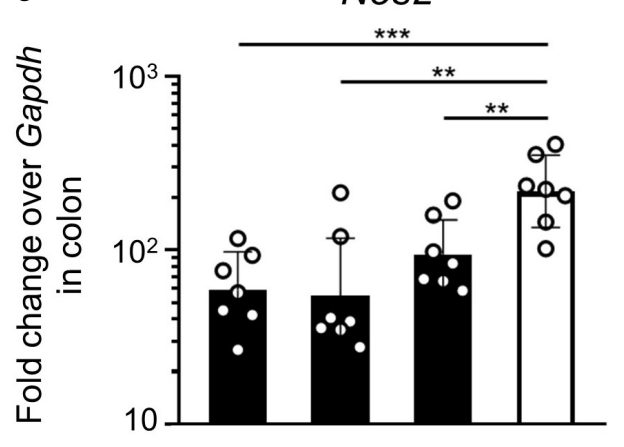

E

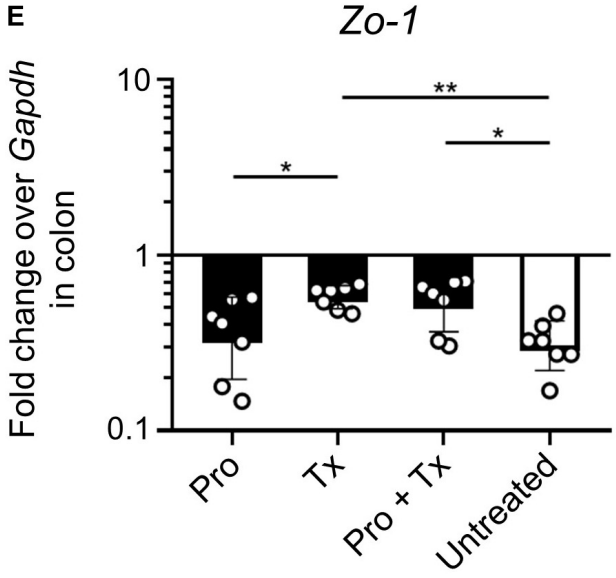

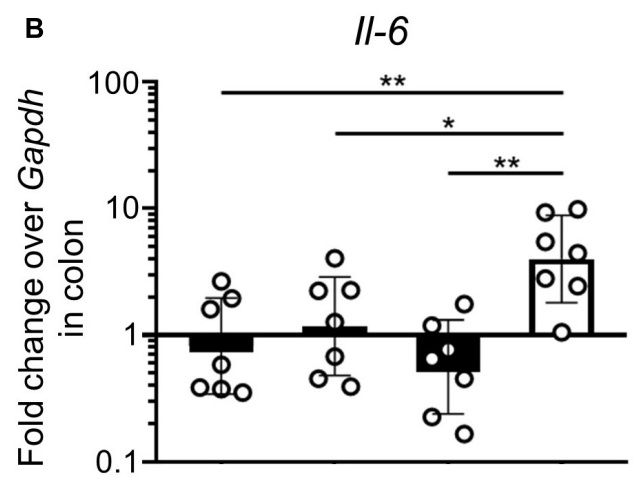

D Ifng

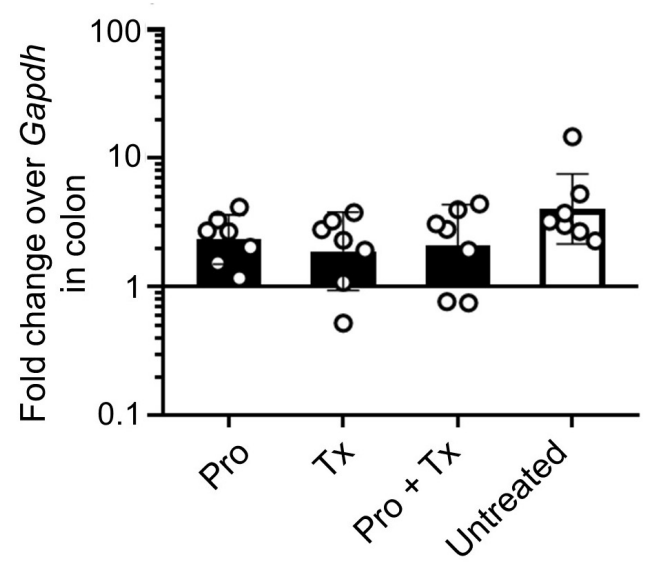

FIGURE 4 | Anti-inflammatory effect of $L$. reuteri KUB-AC5 on the STM-infected mouse colon. At day 4 post infection, mouse colons were collected and quantified for the fold change in mRNA expression of pro-inflammatory cytokines (Kc, II-6, and Ifng), iNOS (Nos2), and tight junction protein (Zo-1) by qPCR. All genes were analyzed with the comparative $C t$ method over the housekeeping gene Gapdh. Bars represent geometric means $\pm \mathrm{SD}$. ${ }^{\star} P<0.05$, ${ }^{\star \star} P<0.01$, and ${ }^{\star \star \star} P<0.001$ compared to all other groups.

for this important foodborne pathogen. STM is a frank pathogen which can be recognized by host gut immune receptors and exploit host responses to its benefit (Baumler et al., 2011; RiveraChavez and Baumler, 2015). By using its virulence factors, a minor population of STM can invade and survive inside host cells while the majority proliferates in the gut lumen of the host (Santos et al., 2009). Winter et al. (2010) showed that
STM conferred a nutritional advantage from gut inflammation, outcompeted resident gut microbiota, and then bloomed in the inflamed gut. Gut inflammation provides several host-derived resources for STM to flourish in the gut. For example, increased oxygen, nitrate, tetrathionate, and lactate levels in the inflamed gut have been reported (Winter et al., 2010; Lopez et al., 2012; Faber et al., 2017; Rogers et al., 2021). These evidences 

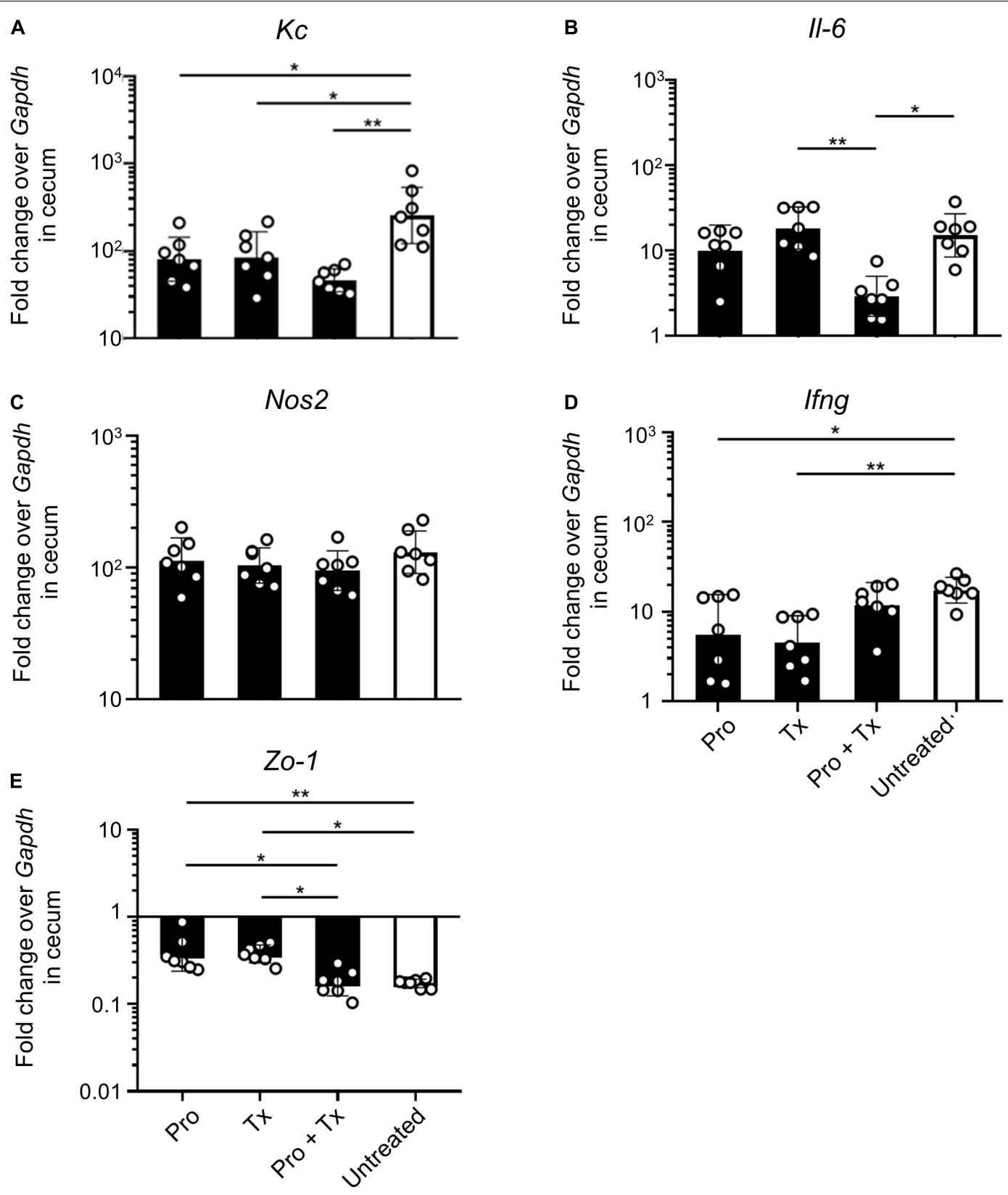

FIGURE 5 | Anti-inflammatory effect of $L$. reuteri KUB-AC5 on the STM-infected mouse cecum. At day 4 post infection, mouse ceca were collected and quantified for the fold change in mRNA expressions of pro-inflammatory cytokines (Kc, Il-6, and lfng), iNOS (Nos2), and tight junction protein (Zo-1) by qPCR. All genes were analyzed with the comparative $C t$ method over the housekeeping gene Gapdh. Bars represent geometric means \pm SD. ${ }^{\star} P<0.05$ and ${ }^{* *} P<0.01$ compared to all other groups.

suggest that the facultative anaerobic bacteria such as STM can harness the host response (gut inflammation) to outcompete the indigenous gut microbiota in the inflamed gut. Hence, strategies that reduce gut inflammation might also decrease STM blooming in the host intestinal tract.

Probiotic microorganisms are a promising way to be used as an alternative to antibiotics in several infectious diseases including an acute NTS (Storr and Stengel, 2021). Two major mechanisms used by probiotics in the inhibition of STM growth have been reviewed (Sassone-Corsi and Raffatellu, 2015). (I) Probiotics could directly inhibit growth of STM by adhesion exclusion, competing for nutrient sources of antimicrobial peptide production. (II) Some probiotics confer an indirect (immunomodulation) effect on gut immunity such as increasing gut barrier integrity and enhancing production of anti-inflammatory cytokines or protective SIgA antibody against STM. The anti-Salmonella effect of probiotic L. reuteri KUBAC5 has been recently reported (Nakphaichit et al., 2019). 
A

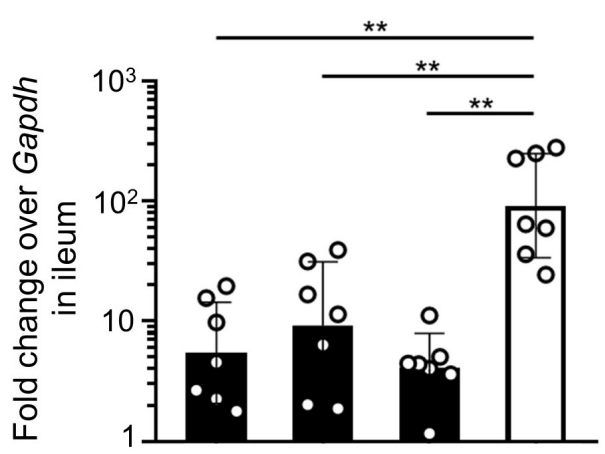

C
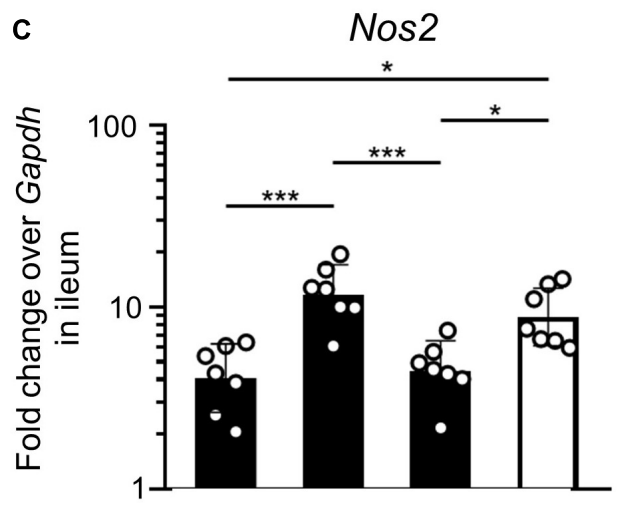

E

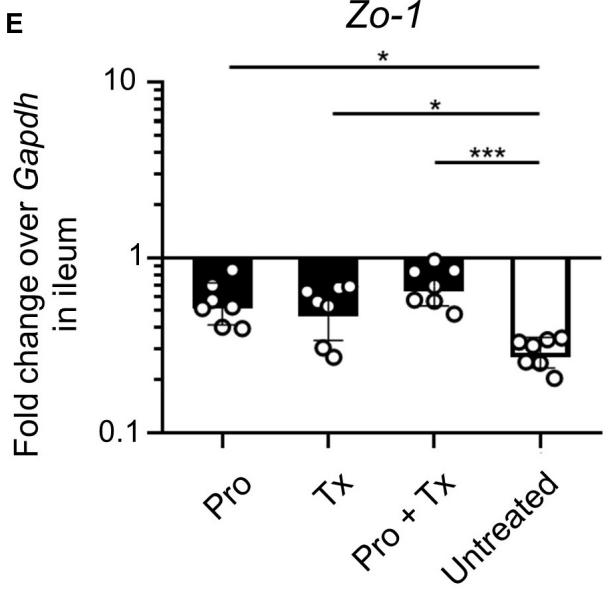

B

II-6

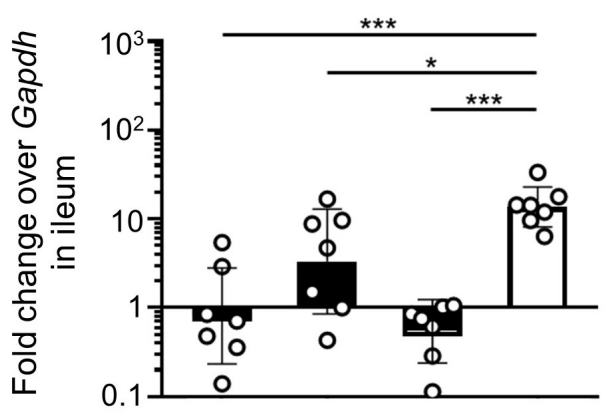

D

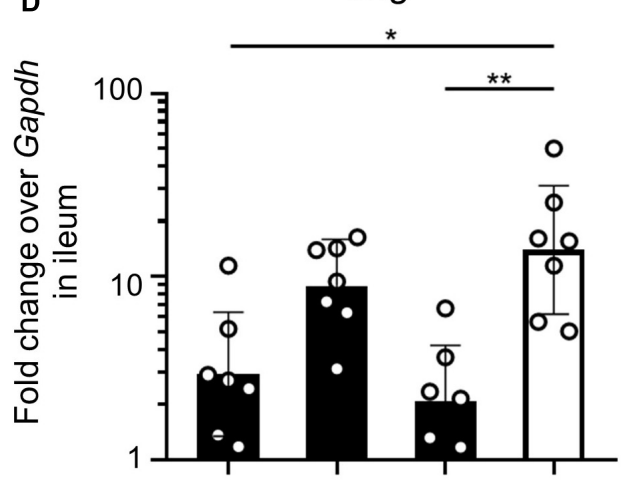

$p^{\circ}$

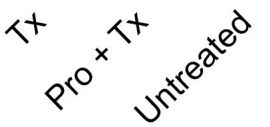

FIGURE 6 | Anti-inflammatory effect of $L$. reuteri KUB-AC5 on the STM-infected mouse ileum. At day 4 post infection, mouse ilea were collected and quantified for the fold change in mRNA expressions of pro-inflammatory cytokines (Kc, II-6, and Ifng), iNOS (Nos2), and tight junction protein (Zo-1) by qPCR. All genes were analyzed with the comparative $C t$ method over the housekeeping gene Gapdh. Bars represent geometric means \pm SD. ${ }^{\star} P<0.05,{ }^{\star \star} P<0.01$, and ${ }^{\star \star \star} P<0.001$ compared to all other groups.

Nakphaichit et al. (2019) showed that high dose AC5 consumption for 3 days prevents Salmonella infection in chickens. The gut microbiota of chickens supplemented with AC5 was altered by causing an increase in Lactobacillaceae and a decrease in Enterobacteriaceae in their ilea and ceca. These results indicate the gut microbiota modulation effect of AC5 on Salmonella infection in chickens. Although the anti-inflammatory effect of probiotic Limosilactobacillus on Salmonella in other experimental models has already been investigated (Abhisingha et al., 2018; Mohanty et al., 2019; Shi et al., 2019; Smialek et al., 2019; Acurcio et al., 2020; Kanmani and Kim, 2020; Kowalska et al., 2020; Mizuno et al., 2020), the strain-specific effect of the probiotic plays a crucial role in their different outcomes. 


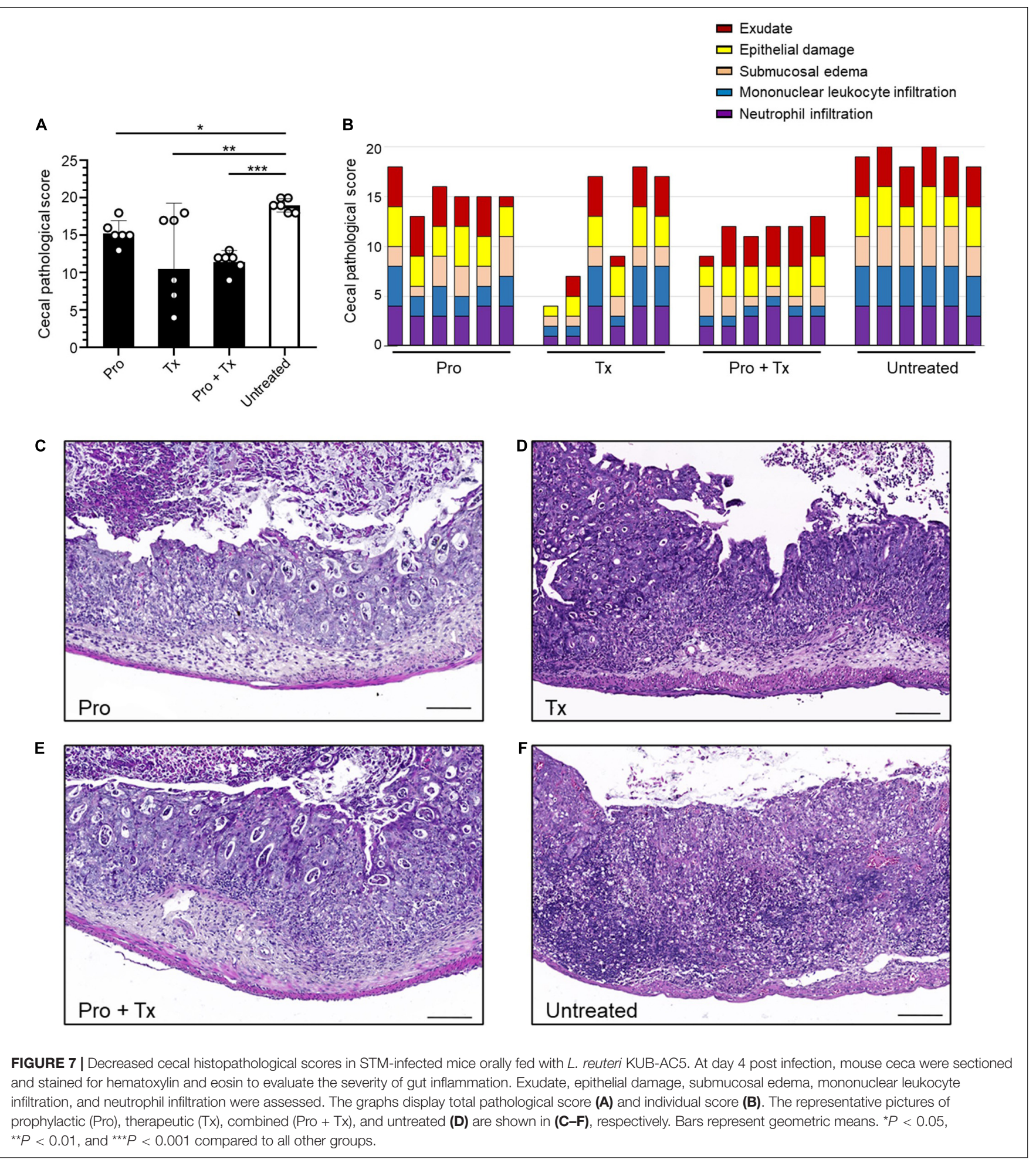

Liu et al. (2018) showed that prophylactic oral feeding with L. plantarum and L. rhamnosus reduced Salmonella numbers and improved gut barrier integrity in C57BL/6 mice. Reduced translocating Salmonella numbers were found in the spleen and liver of L. rhamnosus HN001 orally fed BALB/c mice
(Gill et al., 2001). Interestingly, L. casei CRL431 enhanced the production of IFN- $\gamma$ levels in immune cells isolated from Peyer's patch of mice infected with STM. Castillo et al. (2011) found that the combined effect (Pro $+\mathrm{Tx}$ ) resulted in increased IFN $-\gamma$ and IL-6 levels in mouse intestinal fluid. Nonetheless, most of the 

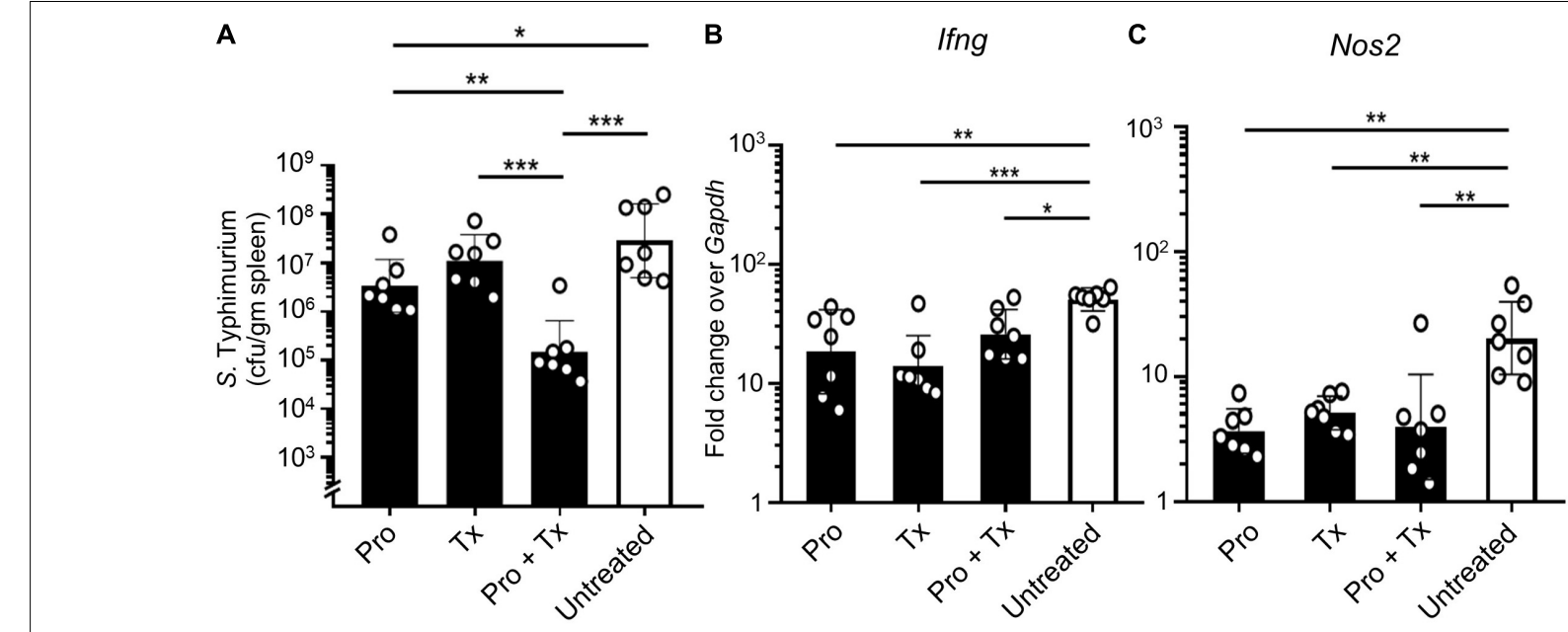

c Nos2

FIGURE 8 | Probiotic L. reuteri KUB-AC5 attenuates a systemic dissemination of STM. AC5-fed mice were divided into three groups (Pro, Tx, and Pro + Tx, black bar) and one untreated group (white bar). Mouse spleens were harvested at day 4 post infection and homogenized for STM cfu/gm determination (A). Fold change of Ifng and Nos2 genes in mouse spleen was calculated by qPCR (B,C, respectively). All genes were analyzed with the comparative Ct method over the housekeeping gene Gapdh. Bars represent geometric means $\pm \mathrm{SD}$. ${ }^{\star} P<0.05,{ }^{\star \star} P<0.01$, and ${ }^{\star \star \star} P<0.001$ compared to all other groups.

previous studies into the probiotic effect of Limosilactobacillus against STM were performed in a non-robust gut inflammation mouse typhoid model. Here, we used a streptomycin-pretreated mouse colitis model to investigate anti-Salmonella and antiinflammatory effects of $L$. reuteri KUB-AC5.

The direct anti-Salmonella effect of AC5 is shown in Figure 1. We investigated the direct antagonistic effects of AC5 on STM using co-culture growth, spot-on lawn, and agar well-diffusion assays. Our data showed that the direct antagonism of AC5 on STM could arise from either the cells or cell-free components of AC5. These data confirmed the anti-Salmonella activity of AC5 in vitro as previously shown (Nitisinprasert et al., 2000). Previous studies also demonstrated that AC5 inhibited in vitro growth of several strains of Salmonella and Escherichia coli by production of short chain fatty acids and antimicrobial peptides not as a result of the acidity effect. Recently, the antimicrobial peptide KAC5 produced by AC5 was characterized and was shown to have no similarity to bacteriocin (Sobanbua et al., 2020). KAC5 of AC5 demonstrated a broad inhibition spectrum against several Gram-positive and -negative bacteria but not against lactic acid-producing bacteria. Next, we compared the role of prophylactic, therapeutic, and combined (prophylactic and therapeutic) AC5 feeding on STM infection in mice. Our data showed that all groups of AC5-fed mice had decreased STM numbers in several parts of their gut and spleen. Interestingly, AC5 caused a more significant reduced in STM numbers in the colon content than in the cecal content in all groups of treated mice. This suggests that the antagonistic activity of AC5 is gut site-specific on STM in mice. A previous study by Jiang et al. (2019) showed that intraperitoneal administration of L. reuteri ATCC 55730 (both live and heat-inactivated forms) reduced severity of an invasive NTS in mice by macrophage activation. In this study, we demonstrated the role of oral consumption of L. reuteri KUB AC-5 in both prevention and treatment of an acute NTS. The combined feeding (Pro + Tx) of AC5 resulted in the highest anti-Salmonella effect in the mouse gut lumen (gut contents) compared to the other feeding approaches. These data suggest a positive role of AC5 feeding and its duration on the inhibition of STM proliferation and invasion in the gut of mice.

Previous studies revealed that AC5 predominated in the ileum and cecum of chicken (Nakphaichit et al., 2011; Sobanbua et al., 2019). Interestingly, our data showed that AC5 could be detected in mouse colon, cecum, and ileum with the highest numbers in the ileum (Figure 3). Mice in the combined group also had the highest amount of AC5 compared to that of the therapeutic and prophylactic groups. Mice in the prophylactic group had the lowest amount of AC5 in their colon, cecum, and ileum. These results indicated that AC5 can transiently colonize the gut of mice and is dependent on feeding duration.

Next, we investigated the anti-inflammatory activity of AC5 on STM infection. Non-typhoidal Salmonella induces a robust gut inflammation in several agricultural animal hosts including cattle, swine, and poultry (Fasina et al., 2008; Bai et al., 2014; Yu et al., 2017; Dar et al., 2019; Huang et al., 2019). However, to date there are few reports on the in vivo gut inflammatory attenuating effect of probiotic Limosilactobacillus (Yu et al., 2017; Pradhan et al., 2019; Peng et al., 2020). Our data showed that AC5 reduced the gene expression of pro-inflammatory cytokine $(K c$, Il-6, and Ifng) and Nos 2 in mouse colon, cecum, and ileum. The tight junction protein gene $(\mathrm{Zo}-1)$ expression was upregulated in the gut of AC5-fed mice (Figures 4-7). However, different gene expressions were observed in different sites of mouse gut. The results from a prophylactic group of mice (fed with AC5 for 7 days before STM infection) could indicate, at least in part, an indirect inhibitory growth effect of AC5 on STM (Supplementary Figure 1). Our data show that numbers of STM in the gut lumen of the prophylactic group were reduced in comparison to that of the therapeutic group (Figures 2A,B), while AC5 can 
transiently colonize mouse gut (Figure 3). However, the degree of gut inflammation is also depending on the numbers of STM in the gut lumen, and vice versa (Winter et al., 2010). By enhancing the nutrient advantage for facultative anaerobes in the inflamed gut lumen, the reduced gut inflammation could come from a direct inhibitory effect of AC5 on STM in the gut lumen as well. Surprisingly, there was no statistically significant difference in the attenuation of gut inflammation between groups of AC5-fed mice. Reduced gut inflammation by AC5 feeding might be due to the decrease in STM proliferation by limiting their necessary resources such as host-derived nutrients. In addition, AC5 prevented the systemic dissemination of STM from the gut into spleen in this model perhaps due to the luminal STM population in the gut (Figure 8). The lowest disseminated numbers of STM in spleen were found in the combined group. This suggested that the beneficial role of AC5 in an invasive NTS may come from either the duration of feeding of AC5 or an additional effect of direct and indirect colonization resistance of AC5. However, a mouse typhoid model (without antibiotic pretreatment) should be used for further investigation into whether AC5 attenuates the severity of systemic disease caused by Salmonella.

\section{CONCLUSION}

Oral administration of the probiotic L. reuteri KUB-AC5 reduced the severity of acute NTS in a mouse model. In vivo antiSalmonella (decreasing numbers of STM) and anti-inflammatory (attenuation of gut and spleen inflammation) effects of AC5 have been revealed. AC5 is more effective against STM when given as a combination (prophylactic and therapeutic) indicating the roles of feeding duration together with the combinatorial effects (direct and indirect colonization resistance) on the probiotic activities of AC5. However, other possible anti-inflammatory mechanisms of AC5, for example, the activation of immunosuppressive regulatory $\mathrm{T}$ cells, production of gut mucosal SIgA antibody, or alteration in gut microbial metabolites (e.g., short-chain fatty acid levels) are required further investigations.

\section{REFERENCES}

Abhisingha, M., Dumnil, J., and Pitaksutheepong, C. (2018). 'Selection of Potential Probiotic Lactobacillus with Inhibitory Activity Against Salmonella and Fecal Coliform Bacteria'. Probiotics Antimicrob. Proteins 10, 218-227. doi: 10.1007/ s12602-017-9304-8

Acurcio, L. B., Wuyts, S., de Cicco Sandes, S. H., Sant'anna, F. M., Bastos, R. W., Reis, D. C. Dos, et al. (2020). Milk Fermented by Lactobacillus paracasei NCC 2461 (ST11) Modulates the Immune Response and Microbiota to Exert its Protective Effects Against Salmonella typhimurium Infection in Mice. Probiotics Antimicrob. Proteins 12, 1398-1408. doi: 10.1007/s12602-020-09634-X

Adetoye, A., Pinloche, E., Adeniyi, B. A., and Ayeni, F. A. (2018). 'Characterization and anti-salmonella activities of lactic acid bacteria isolated from cattle faeces'. BMC Microbiol. 18:96.

Antunes, P., Mourão, J., Campos, J., and Peixe, L. (2016). 'Salmonellosis: the role of poultry meat'. Clin. Microbiol. Infect. 22, 110-121. doi: 10.1016/j.cmi.2015.12. 004

Bai, S. P., Huang, Y., Luo, Y. H., Wang, L. L., Ding, X. M., Wang, J. P., et al. (2014). 'Alteration in lymphocytes responses, cytokine and chemokine profiles in laying

\section{DATA AVAILABILITY STATEMENT}

This work was supported by the Faculty of Medicine Research Fund (MIC-2561-05411), Chiang Mai University, Chiang Mai, Thailand, and the Thailand Research Fund (MRG-6180187) (PT).

\section{ETHICS STATEMENT}

The animal study was reviewed and approved by the Animal Care and Use Committee, Chiang Mai University, Thailand in accordance with the Association for Assessment and Accreditation of Laboratory Animal Care (AAALAC) guidelines (Approval No. 2559/MC-0005).

\section{AUTHOR CONTRIBUTIONS}

PT generated the research. PT and SB designed and conceptualized the research. SB, CS, MN, and PT performed the research. SN provided the probiotic L. reuteri KUB AC5 and supervised the study. TK performed the histopathological study. $\mathrm{SB}, \mathrm{MN}$, and PT analyzed the data. PT, SB, KN, and MN wrote and reviewed the manuscript. All authors contributed to the article and approved the submitted version.

\section{FUNDING}

This work was supported by the Faculty of Medicine Research Fund (MIC-2561-05224), Chiang Mai University, Chiang Mai, Thailand, and the Thailand Research Fund (MRG-6180187) (PT).

\section{SUPPLEMENTARY MATERIAL}

The Supplementary Material for this article can be found online at: https://www.frontiersin.org/articles/10.3389/fmicb. 2021.716761/full\#supplementary-material

hens infected with Salmonella Typhimurium'. Vet. Immunol. Immunopathol. 160, 235-243. doi: 10.1016/j.vetimm.2014.05.015

Balasubramanian, R., Im, J., Lee, J. S., Jeon, H. J., Mogeni, O. D., Kim, J. H., et al. (2019). 'The global burden and epidemiology of invasive non-typhoidal Salmonella infections'. Hum. Vaccin. Immunother. 15, 1421-1426. doi: 10.1080/ 21645515.2018.1504717

Barman, M., Unold, D., Shifley, K., Amir, E., Hung, K., Bos, N., et al. (2008). 'Enteric salmonellosis disrupts the microbial ecology of the murine gastrointestinal tract'. Infect. Immun. 76, 907-915. doi: 10.1128/iai.01432-07

Barthel, M., Hapfelmeier, S., Quintanilla-Martinez, L., Kremer, M., Rohde, M., Hogardt, M., et al. (2003). 'Pretreatment of mice with streptomycin provides a Salmonella enterica serovar Typhimurium colitis model that allows analysis of both pathogen and host'. Infect. Immun. 71, 2839-2858. doi: 10.1128/iai.71. 5.2839-2858.2003

Baumler, A. J., Winter, S. E., Thiennimitr, P., and Casadesus, J. (2011). 'Intestinal and chronic infections: Salmonella lifestyles in hostile environments'. Environ. Microbiol. Rep. 3, 508-517. doi: 10.1111/j.1758-2229.2011.00242.x

Bernad-Roche, M., Casanova-Higes, A., Marín-Alcal, C. M., Cebollada-Solanas, A., and Mainar-Jaime, R. C. (2021). Salmonella Infection in Nursery Piglets and Its 
Role in the Spread of Salmonellosis to Further Production Periods. Pathogens 2021:10.

Bohnhoff, M., Drake, B. L., and Miller, C. P. (1954). 'Effect of streptomycin on susceptibility of intestinal tract to experimental Salmonella infection'. Proc. Soc. Exp. Biol. Med. 86, 132-137. doi: 10.3181/00379727-86-21030

Bohnhoff, M., and Miller, C. P. (1962). 'Enhanced susceptibility to Salmonella infection in streptomycin-treated mice'. J. Infect. Dis. 111, 117-127. doi: 10. 1093/infdis/111.2.117

Broz, P., and Monack, D. M. (2011). 'Molecular mechanisms of inflammasome activation during microbial infections'. Immunol. Rev. 243, 174-190. doi: 10. 1111/j.1600-065x.2011.01041.x

Broz, P., Ohlson, M. B., and Monack, D. M. (2012). 'Innate immune response to Salmonella typhimurium, a model enteric pathogen'. Gut. Microbes 3, 62-70. doi: 10.4161/gmic.19141

Campos, J., Mourão, J., Peixe, L., and Antunes, P. (2019). Non-typhoidal Salmonella in the Pig Production Chain: A Comprehensive Analysis of Its Impact on Human Health. Pathogens 2019:8.

Castanon, J. I. (2007). 'History of the use of antibiotic as growth promoters in European poultry feeds'. Poult. Sci. 86, 2466-2471. doi: 10.3382/ps.2007-00249

Castillo, N. A., Perdigón, G., and de Moreno de Leblanc, A. (2011). Oral administration of a probiotic Lactobacillus modulates cytokine production and TLR expression improving the immune response against Salmonella enterica serovar Typhimurium infection in mice. BMC Microbiol. 11:177. doi: 10.1186/ 1471-2180-11-177

Castro-Vargas, R. E., Herrera-Sánchez, M. P., Rodríguez-Hernández, R., and Rondón-Barragán, I. S. (2020). 'Antibiotic resistance in Salmonella spp. isolated from poultry: A global overview'. Vet. World 13, 2070-2084. doi: 10.14202/ vetworld.2020.2070-2084

Chen, Y., Liu, H., Chen, M., Sun, H. Y., and Wu, Y. N. (2020). 'The human health burden of non-typhoidal Salmonella enterica and Vibrio parahaemolyticus foodborne gastroenteritis in Shanghai, east China'. PLoS One 15:e0242156. doi: 10.1371/journal.pone.0242156

Dar, M. A., Urwat, U., Ahmad, S. M., Ahmad, R., Kashoo, Z. A., Dar, T. A., et al. (2019). 'Gene expression and antibody response in chicken against Salmonella Typhimurium challenge'. Poult. Sci. 98, 2008-2013. doi: 10.3382/ps/pey560

Drago, L., Gismondo, M. R., Lombardi, A., de Haën, C., and Gozzini, L. (1997). 'Inhibition of in vitro growth of enteropathogens by new Lactobacillus isolates of human intestinal origin'. FEMS Microbiol. Lett. 153, 455-463. doi: 10.1111/ j.1574-6968.1997.tb12610.x

Faber, F., Thiennimitr, P., Spiga, L., Byndloss, M. X., Litvak, Y., Lawhon, S., et al. (2017). 'Respiration of Microbiota-Derived 1,2-propanediol Drives Salmonella Expansion during Colitis'. PLoS Pathog. 13:e1006129. doi: 10.1371/journal. ppat. 1006129

Fang, H. W., Fang, S. B., Chiang Chiau, J. S., Yeung, C. Y., Chan, W. T., Jiang, C. B., et al. (2010). 'Inhibitory effects of Lactobacillus casei subsp. rhamnosus on Salmonella lipopolysaccharide-induced inflammation and epithelial barrier dysfunction in a co-culture model using Caco-2/peripheral blood mononuclear cells'. J. Med. Microbiol. 59, 573-579. doi: 10.1099/jmm.0.009662-0

Fasina, Y. O., Holt, P. S., Moran, E. T., Moore, R. W., Conner, D. E., and McKee, S. R. (2008). 'Intestinal cytokine response of commercial source broiler chicks to Salmonella typhimurium infection'. Poult. Sci. 87, 1335-1346. doi: 10.3382/ ps.2007-00526

Ferrari, R. G., Rosario, D. K. A., Cunha-Neto, A., Mano, S. B., Figueiredo, E. E. S., and Conte-Junior, C. A. (2019). Worldwide Epidemiology of Salmonella Serovars in Animal-Based Foods: a Meta-analysis. Appl. Environ. Microbiol. 2019:85.

Gill, H. S., Shu, Q., Lin, H., Rutherfurd, K. J., and Cross, M. L. (2001). 'Protection against translocating Salmonella typhimurium infection in mice by feeding the immuno-enhancing probiotic Lactobacillus rhamnosus strain HN001'. Med. Microbiol. Immunol. 190, 97-104. doi: 10.1007/s004300100095

Gordon, M. A., Banda, H. T., Gondwe, M., Gordon, S. B., Boeree, M. J., Walsh, A. L., et al. (2002). 'Non-typhoidal salmonella bacteraemia among HIV-infected Malawian adults: high mortality and frequent recrudescence'. Aids 16, 16331641. doi: 10.1097/00002030-200208160-00009

Hai, D., Kong, L. Y., Lu, Z. X., Huang, X. Q., and Bie, X. M. (2021). Inhibitory effect of different chicken-derived lactic acid bacteria isolates on drug resistant Salmonella SE47 isolated from eggs. Lett. Appl. Microbiol. 73, 54-63. doi: 10. 1111/lam.13475
Hausmann, A., and Hardt, W. D. (2019). The Interplay between Salmonella enterica Serovar Typhimurium and the Intestinal Mucosa during Oral Infection. Microbiol. Spectr. 2019:7.

Heredia, N., and García, S. (2018). 'Animals as sources of food-borne pathogens: A review'. Anim. Nutr. 4, 250-255. doi: 10.1016/j.aninu.2018.04.006

Huang, K., Fresno, A. H., Skov, S., and Olsen, J. E. (2019). 'Dynamics and Outcome of Macrophage Interaction Between Salmonella Gallinarum, Salmonella Typhimurium, and Salmonella Dublin and Macrophages From Chicken and Cattle. Front. Cell Infect. Microbiol. 9:420.

Jia, D., Wang, Y., Wang, J., Liu, J., Li, H., Liu, A., et al. (2021). Lactobacillus animalis pZL8a: a potential probiotic isolated from pig feces for further research. 3 Biotech 11:132.

Jiang, P., Yang, W., Jin, Y., Huang, H., Shi, C., Jiang, Y., et al. (2019). 'Lactobacillus reuteri protects mice against Salmonella typhimurium challenge by activating macrophages to produce nitric oxide'. Microb. Pathog. 137:103754. doi: 10. 1016/j.micpath.2019.103754

Kakabadze, E., Grdzelishvili, N., Sanikidze, L., Makalatia, K., and Chanishvili, N. (2020). Revival of microbial therapeutics, with emphasis on probiotic lactobacillus (REVIEW). Georgian Med. News 2020, 129-134.

Kanmani, P., and Kim, H. (2020). 'Beneficial effect of immunobiotic strains on attenuation of Salmonella induced inflammatory response in human intestinal epithelial cells'. PLoS One 15:e229647. doi: 10.1371/journal.pone.022 9647

Kim, B. S., Yun, C. H., Han, S. H., Song, K. D., and Kang, S. S. (2021). 'Inhibitory Effect of Lipoteichoic Acid Derived from Three Lactobacilli on FlagellinInduced IL-8 Production in Porcine Peripheral Blood Mononuclear Cells'. Probiot. Antimicrob. Proteins 13, 72-79. doi: 10.1007/s12602-020-09682-3

Kongsanan, P., Angkititrakul, S., Kiddee, A., and Tribuddharat, C. (2020). Spread of antimicrobial resistant Salmonella from poultry to humans in Thailand. Jpn J. Infect. Dis. 74, 220-227. doi: 10.7883/yoken.jjid.2020.548

Kowalska, J. D., Nowak, A., Śliżewska, K., Stańczyk, M., Łukasiak, M., and Dastych, J. (2020). 'Anti-Salmonella Potential of New Lactobacillus Strains with the Application in the Poultry Industry'. Pol. J. Microbiol. 69, 5-18. doi: 10.33073/ pjm-2020-001

Lima, E. T., Andreatti Filho, R. L., Okamoto, A. S., Noujaim, J. C., Barros, M. R., and Crocci, A. J. (2007). 'Evaluation in vitro of the antagonistic substances produced by Lactobacillus spp. isolated from chickens'. Can J. Vet. Res. 71, 103-107.

Liu, J., Gu, Z., Song, F., Zhang, H., Zhao, J., and Chen, W. (2019). 'Lactobacillus plantarum ZS2058 and Lactobacillus rhamnosus GG Use Different Mechanisms to Prevent Salmonella Infection in vivo'. Front. Microbiol. 10:299.

Liu, J., Hu, D., Chen, Y., Huang, H., Zhang, H., Zhao, J., et al. (2018). 'Strain-specific properties of Lactobacillus plantarum for prevention of Salmonella infection'. Food Funct. 9, 3673-3682.

Lopez, C. A., Rivera-Chavez, F., Byndloss, M. X., and Baumler, A. J. (2015). 'The Periplasmic Nitrate Reductase NapABC Supports Luminal Growth of Salmonella enterica Serovar Typhimurium during Colitis'. Infect. Immun. 83, 3470-3478. doi: 10.1128/iai.00351-15

Lopez, C. A., Winter, S. E., Rivera-Chavez, F., Xavier, M. N., Poon, V., Nuccio, S. P., et al. (2012). Phage-mediated acquisition of a type III secreted effector protein boosts growth of salmonella by nitrate respiration. mBio 2012:3.

Lupp, C., Robertson, M. L., Wickham, M. E., Sekirov, I., Champion, O. L., Gaynor, E. C., et al. (2007). 'Host-mediated inflammation disrupts the intestinal microbiota and promotes the overgrowth of Enterobacteriaceae'. Cell Host Microbe 2, 119-129. doi: 10.1016/j.chom.2007.06.010

Majowicz, S. E., Musto, J., Scallan, E., Angulo, F. J., Kirk, M., O’Brien, S. J., et al. (2010). 'The global burden of nontyphoidal Salmonella gastroenteritis'. Clin. Infect. Dis. 50, 882-889.

Mao, Y., Zeineldin, M., Usmani, M., Uprety, S., Shisler, J. L., Jutla, A., et al. (2021). Distribution and Antibiotic Resistance Profiles of Salmonella enterica in Rural Areas of North Carolina After Hurricane Florence in 2018. Geohealth 5:e2020GH000294.

McLaughlin, P. A., Bettke, J. A., Tam, J. W., Leeds, J., Bliska, J. B., Butler, B. P., et al. (2019). 'Inflammatory monocytes provide a niche for Salmonella expansion in the lumen of the inflamed intestine’. PLoS Pathog. 15:e1007847. doi: 10.1371/ journal.ppat.1007847

Mizuno, H., Arce, L., Tomotsune, K., Albarracin, L., Funabashi, R., Vera, D., et al. (2020). 'Lipoteichoic Acid Is Involved in the Ability of the Immunobiotic Strain 
Lactobacillus plantarum CRL1506 to Modulate the Intestinal Antiviral Innate Immunity Triggered by TLR3 Activation'. Front. Immunol. 11:571.

Mohanty, D., Panda, S., Kumar, S., and Ray, P. (2019). 'In vitro evaluation of adherence and anti-infective property of probiotic Lactobacillus plantarum DM 69 against Salmonella enterica'. Microb. Pathog. 126, 212-217. doi: 10.1016/j. micpath.2018.11.014

Morelli, L., and Capurso, L. (2012). 'FAO/WHO guidelines on probiotics: 10 years later'. J. Clin. Gastroenterol. 46(Suppl.), S1-S2.

Nakphaichit, M., Sobanbua, S., Siemuang, S., Vongsangnak, W., Nakayama, J., and Nitisinprasert, S. (2019). 'Protective effect of Lactobacillus reuteri KUB-AC5 against Salmonella Enteritidis challenge in chickens'. Benef Microbes 10, 43-54. doi: 10.3920/bm2018.0034

Nakphaichit, M., Thanomwongwattana, S., Phraephaisarn, C., Sakamoto, N., Keawsompong, S., Nakayama, J., et al. (2011). 'The effect of including Lactobacillus reuteri KUB-AC5 during post-hatch feeding on the growth and ileum microbiota of broiler chickens'. Poultr. Sci. 90, 2753-2765. doi: 10.3382/ ps.2011-01637

Nitisinprasert, S., Veeraphol, N., Phunjai, B., Prakit, S., Katsumi, D., and Kenji, S. (2000). Screening and Identification of Effective Thermotolerant Lactic Acid Bacteria Producing Antimicrobial Activity Against Escherichia coli and Salmonella sp. Resistant to Antibiotics. Kasetsart J. 2000, 387-400.

Peng, M., Tabashsum, Z., Patel, P., Bernhardt, C., Biswas, C., Meng, J., et al. (2020). 'Prevention of enteric bacterial infections and modulation of gut microbiota with conjugated linoleic acids producing Lactobacillus in mice'. Gut Microbes 11, 433-452. doi: 10.1080/19490976.2019.1638724

Petrova, M. I., Reid, G., and Ter Haar, J. A. (2021). Lacticaseibacillus rhamnosus GR-1, a.k.a. Lactobacillus rhamnosus GR-1: Past and Future Perspectives. Trends Microbiol. 29, 747-761. doi: 10.1016/j.tim.2021.03.010

Pradhan, B., Guha, D., Naik, A. K., Banerjee, A., Tambat, S., Chawla, S., et al. (2019). 'Probiotics L. acidophilus and B. clausii Modulate Gut Microbiota in Th1- and Th2-Biased Mice to Ameliorate Salmonella Typhimurium-Induced Diarrhea'. Probiotics Antimicrob. Proteins 11, 887-904. doi: 10.1007/s12602-018-9 436-5

Preziosi, M. J., Kandel, S. M., Guiney, D. G., and Browne, S. H. (2012). 'Microbiological analysis of nontyphoidal Salmonella strains causing distinct syndromes of bacteremia or enteritis in HIV/AIDS patients in San Diego, California. J. Clin. Microbiol. 50, 3598-3603. doi: 10.1128/jcm.00795-12

Rivera-Chavez, F., and Baumler, A. J. (2015). 'The Pyromaniac Inside You: Salmonella Metabolism in the Host Gut'. Annu. Rev. Microbiol. 69, 31-48. doi: 10.1146/annurev-micro-091014-104108

Rogers, A. W. L., Tsolis, R. M., and Bäumler, A. J. (2021). Salmonella versus the Microbiome. Microbiol. Mol. Biol. Rev. 2021:85.

Santos, R. L. (2014). 'Pathobiology of salmonella, intestinal microbiota, and the host innate immune response'. Front. Immunol. 5:252.

Santos, R. L., Raffatellu, M., Bevins, C. L., Adams, L. G., Tukel, C., Tsolis, R. M., et al. (2009). 'Life in the inflamed intestine. Salmonella style. Trends Microbiol. 17, 498-506. doi: 10.1016/j.tim.2009.08.008

Santos, R. L., Zhang, S., Tsolis, R. M., Kingsley, R. A., Adams, L. G., and Baumler, A. J. (2001). 'Animal models of Salmonella infections: enteritis versus typhoid fever'. Microbes Infect. 3, 1335-1344. doi: 10.1016/s1286-4579(01)01 495-2

Sargun, A., Sassone-Corsi, M., Zheng, T., Raffatellu, M., and Nolan, E. M. (2021). Conjugation to Enterobactin and Salmochelin S4 Enhances the Antimicrobial Activity and Selectivity of $\beta$-Lactam Antibiotics against Nontyphoidal Salmonella. ACS Infect. Dis. 7, 1248-1259. doi: 10.1021/acsinfecdis.1c0 0005

Sarichai, P., Buddhasiri, S., Walters, G. E., Khantawa, B., Kaewsakhorn, T., Chantarasakha, K., et al. (2020). 'Pathogenicity of clinical Salmonella enterica serovar Typhimurium isolates from Thailand in a mouse colitis model'. Microbiol. Immunol. 64, 679-693. doi: 10.1111/1348-0421.12837

Sassone-Corsi, M., and Raffatellu, M. (2015). 'No vacancy: how beneficial microbes cooperate with immunity to provide colonization resistance to pathogens'. J. Immunol. 194, 4081-4087. doi: 10.4049/jimmunol.1403169

Schmittgen, T. D., and Livak, K. J. (2008). 'Analyzing real-time PCR data by the comparative C(T) method'. Nat. Protoc. 3, 1101-1108. doi: 10.1038/nprot. 2008.73

Sedrakyan, A. M., Ktsoyan, Z. A., Arakelova, K. A., Zakharyan, M. K., Hovhannisyan, A. I., Gevorgyan, Z. U., et al. (2020).
'Extended-Spectrum $\beta$-Lactamases in Human Isolates of MultidrugResistant Non-typhoidal Salmonella enterica'. Front. Microbiol. 11:59 2223.

Shi, S., Qi, Z., Sheng, T., Tu, J., Shao, Y., and Qi, K. (2019). 'Antagonistic trait of Lactobacillus reuteri S5 against Salmonella enteritidis and assessment of its potential probiotic characteristics'. Microb. Pathog. 137:103773. doi: 10.1016/j. micpath.2019.103773

Smialek, M., Kaczorek, E., Szczucińska, E., Burchardt, S., Kowalczyk, J., Tykałowski, B., et al. (2019). 'Evaluation of Lactobacillus spp. and yeast based probiotic (Lavipan) supplementation for the reduction of Salmonella Enteritidis after infection of broiler chickens'. Pol. J. Vet. Sci. 22, 5-10.

Sobanbua, S., Dolkittikul, S., Nakphaichit, M., Keawsompong, S., and Nitisinprasert, S. (2019). 'Antimicrobial peptide presenting potential strain-specific real time polymerase chain reaction assay for detecting the probiotic Lactobacillus reuteri KUB-AC5 in chicken intestine'. Poult. Sci. 99, 526-535. doi: 10.3382/ps/pez 549

Sobanbua, S., Tangthong, J., Suveatwatanakul, A., Nakphaichit, M., Keawsompong, S., and Nitisinprasert, S. (2020). Cloning and expression of the antimicrobial peptide from Lactobacillus reuteri KUB-AC5 and its characterization. Internat. J. Agricult. Technol. 2020, 1013-1036.

Stecher, B., Robbiani, R., Walker, A. W., Westendorf, A. M., Barthel, M., Kremer, M., et al. (2007). 'Salmonella enterica serovar typhimurium exploits inflammation to compete with the intestinal microbiota'. PLoS Biol. 5:21772189.

Stojiljkovic, I., Baumler, A. J., and Heffron, F. (1995). 'Ethanolamine utilization in Salmonella typhimurium: nucleotide sequence, protein expression, and mutational analysis of the $\operatorname{cch} A \operatorname{cchB}$ eutE eut $\mathrm{eutG}$ eut $\mathrm{H}$ gene cluster'. J. Bacteriol. 177, 1357-1366. doi: 10.1128/jb.177.5.1357-1366. 1995

Storr, M., and Stengel, A. (2021). Systematic review: clinical evidence of probiotics in the prevention of antibiotic-associated diarrhoea. MMW Fortschr. Med 163, $19-26$.

Sun, F., Li, X., Wang, Y., Wang, F., Ge, H., Pan, Z., et al. (2021). 'Epidemic patterns of antimicrobial resistance of Salmonella enterica serovar Gallinarum biovar Pullorum isolates in China during the past half-century'. Poult. Sci. 100:100894. doi: 10.1016/j.psj.2020.12.007

Tasmin, R., Gulig, P. A., and Parveen, S. (2019). 'Detection of Virulence PlasmidEncoded Genes in Salmonella Typhimurium and Salmonella Kentucky Isolates Recovered from Commercially Processed Chicken Carcasses'. J. Food Prot. 82, 1364-1368. doi: 10.4315/0362-028x.jpp-18-552

Thiennimitr, P., Winter, S. E., and Bäumler, A. J. (2012). 'Salmonella, the host and its microbiota'. Curr. Opin. Microbiol. 15, 108-114. doi: 10.1016/j.mib.2011.10. 002

Thiennimitr, P., Winter, S. E., Winter, M. G., Xavier, M. N., Tolstikov, V., Huseby, D. L., et al. (2011). 'Intestinal inflammation allows Salmonella to use ethanolamine to compete with the microbiota'. Proc. Natl. Acad. Sci. U S A 108, 17480-17485. doi: 10.1073/pnas.110785 7108

Tsolis, R. M., Kingsley, R. A., Townsend, S. M., Ficht, T. A., Adams, L. G., and Baumler, A. J. (1999). 'Of mice, calves, and men. Comparison of the mouse typhoid model with other Salmonella infections. Adv. Exp. Med. Biol. 473, 261-274.

Tsolis, R. M., Xavier, M. N., Santos, R. L., and Baumler, A. J. (2011). 'How to become a top model: impact of animal experimentation on human Salmonella disease research'. Infect. Immun. 79, 1806-1814. doi: 10.1128/iai.013 69-10

Ur Rahman, S., Ali, T., Ali, I., Khan, N. A., Han, B., and Gao, J. (2018). The Growing Genetic and Functional Diversity of Extended Spectrum Beta-Lactamases. Biomed. Res. Int. 2018:9519718.

Whistler, T., Sapchookul, P., McCormick, D. W., Sangwichian, O., Jorakate, P., Makprasert, S., et al. (2018). 'Epidemiology and antimicrobial resistance of invasive non-typhoidal Salmonellosis in rural Thailand from 20062014'. PLoS Negl. Trop Dis. 12:e0006718. doi: 10.1371/journal.pntd.000 6718

Winter, S. E., Thiennimitr, P., Winter, M. G., Butler, B. P., Huseby, D. L., Crawford, R. W., et al. (2010). 'Gut inflammation provides a respiratory electron acceptor for Salmonella'. Nature 467, 426-429. 
Winter, S. E., Winter, M. G., Xavier, M. N., Thiennimitr, P., Poon, V., Keestra, A. M., et al. (2013). 'Host-derived nitrate boosts growth of E. coli in the inflamed gut'. Science 339, 708-711. doi: 10.1126/science.123 2467

Yeung, C. Y., Chiang Chiau, J. S., Chan, W. T., Jiang, C. B., Cheng, M. L., Liu, H. L., et al. (2013). 'In vitro prevention of salmonella lipopolysaccharide-induced damages in epithelial barrier function by various lactobacillus strains'. Gastroenterol. Res. Pract. 2013:97 3209.

Yu, J., Zhu, Y. H., Yang, G. Y., Zhang, W., Zhou, D., Su, J. H., et al. (2017). 'Antiinflammatory capacity of Lactobacillus rhamnosus GG in monophasic variant Salmonella infected piglets is correlated with impeding NLRP6-mediated host inflammatory responses'. Vet. Microbiol. 210, 91-100. doi: 10.1016/j.vetmic. 2017.08.008

Zhao, W. H., and Hu, Z. Q. (2013). 'Epidemiology and genetics of CTX$\mathrm{M}$ extended-spectrum $\beta$-lactamases in Gram-negative bacteria'. Crit. Rev. Microbiol. 39, 79-101. doi: 10.3109/1040841x.2012.691460
Conflict of Interest: The authors declare that the research was conducted in the absence of any commercial or financial relationships that could be construed as a potential conflict of interest.

Publisher's Note: All claims expressed in this article are solely those of the authors and do not necessarily represent those of their affiliated organizations, or those of the publisher, the editors and the reviewers. Any product that may be evaluated in this article, or claim that may be made by its manufacturer, is not guaranteed or endorsed by the publisher.

Copyright (C) 2021 Buddhasiri, Sukjoi, Kaewsakhorn, Nambunmee, Nakphaichit, Nitisinprasert and Thiennimitr. This is an open-access article distributed under the terms of the Creative Commons Attribution License (CC BY). The use, distribution or reproduction in other forums is permitted, provided the original author(s) and the copyright owner(s) are credited and that the original publication in this journal is cited, in accordance with accepted academic practice. No use, distribution or reproduction is permitted which does not comply with these terms. 